\title{
Investigation of the mechanism of enhanced skin penetration by ultradeformable liposomes
}

This article was published in the following Dove Press journal:

International Journal of Nanomedicine

25 July 2014

Number of times this article has been viewed

\section{Thirapit Subongkot \\ Boonnada \\ Pamornpathomkul \\ Theerasak Rojanarata \\ Praneet Opanasopit \\ Tanasait Ngawhirunpat}

Faculty of Pharmacy, Silpakorn

University, Nakhon Pathom, Thailand
Abstract: This study aimed to determine the mechanism by which ultradeformable liposomes (ULs) with terpenes enhance skin penetration for transdermal drug delivery of fluorescein sodium, using transmission electron microscopy (TEM) and confocal laser scanning microscopy (CLSM). Skin treated with ULs containing d-limonene, obtained from in vitro skin penetration studies, was examined via TEM to investigate the effect of ULs on ultrastructural changes of the skin, and to evaluate the mechanism by which ULs enhance skin penetration. The receiver medium collected was analyzed by TEM and CLSM to evaluate the mechanism of the drug carrier system. Our findings revealed that ULs could enhance penetration by denaturing intracellular keratin, degrading corneodesmosomes, and disrupting the intercellular lipid arrangement in the stratum corneum. As inferred from the presence of intact vesicles in the receiver medium, ULs are also able to act as a drug carrier system. CLSM images showed that intact vesicles of ULs might penetrate the skin via a transappendageal pathway, potentially a major route of skin penetration.

Keywords: ultradeformable liposomes, mechanism of enhanced skin penetration, transmission electron microscopy, confocal laser scanning microscopy

\section{Introduction}

A transdermal drug delivery system utilizes skin to deliver drug into the circulation system. The main obstacle in this system is poor percutaneous absorption, because the uppermost layer of the skin (the stratum corneum) acts as a barrier. To improve percutaneous absorption of drug, many techniques have been applied, such as iontop horesis, ${ }^{1}$ sonophoresis, ${ }^{2}$ microneedles, ${ }^{3}$ and lipid vesicle carriers. ${ }^{4,5}$ Among the group of lipid vesicle carriers, ultradeformable liposomes (ULs) have received considerable attention in transdermal drug delivery research. Introduced by Cevc and Blume, ${ }^{6} \mathrm{ULs}$ are a type of elastic liposome, created by incorporating edge activators into liposomes. ULs have been shown to be effective for transdermal delivery of macromolecules, which are difficult to permeate through the skin. ${ }^{7-11}$

Two possible mechanisms proposed for the enhancement of skin penetration by ULs are a penetration-enhancing effect and a drug carrier system. The first mechanism suggests that ULs increase drug penetration into the skin by acting as a penetration enhancer. ${ }^{12,13}$ The second mechanism proposes that ULs act as a drug carrier system. ${ }^{6,14-18}$ To investigate the penetration-enhancing effect, various techniques have been used, such as infrared spectroscopy, differential scanning calorimetry, and skin penetration study. Although these techniques proved to be convenient and simple, they could not reveal the ultrastructural changes in skin caused by penetration enhancers. To assess the drug carrier system mechanism, drug-loaded vesicles must be able to pass through the skin into systemic circulation as intact vesicles. Cevc et al ${ }^{15}$ reported the presence of drug-loaded vesicles in the blood of mice after fluorescently-labeled
Correspondence: Tanasait Ngawhirunpat Department of Pharmaceutical

Technology, Faculty of Pharmacy, Silpakorn University, 6 Racchamakkanai Road, Muang, Nakhon Pathom 73000, Thailand

Tel +6634255800

Fax +6634255801

Email tanasait@su.ac.th 
transfersomes were applied to the skin. This finding was evidence that ULs penetrated the skin and entered the systemic circulation as intact vesicles. Regarding in vivo and in vitro experiments of human skin treated with elastic vesicles, Honeywell-Nguyen et $\mathrm{al}^{16}$ suggested that penetration of intact vesicles into viable epidermis is unlikely to happen, because little vesicle material was found in the deepest layers of stratum corneum.

Understanding the mechanism of action of vesicle-skin interaction is a prerequisite for transdermal drug delivery development and optimization. However, the mechanism of action of vesicle-skin interaction has not been clearly investigated. Therefore, we performed an in vitro skin penetration study, using porcine skin treated with ULs to evaluate the penetration enhancement mechanism, focusing on changes in the ultrastructure of skin affected by vesicle interaction. A UL with d-limonene was selected as a candidate to examine both proposed mechanisms. ${ }^{19,20}$ Transmission electron microscopy (TEM) was used to visualize changes in cellular components caused by UL interaction, inside both stratum corneum and viable tissues, in order to assess the penetration-enhancing mechanism. TEM and confocal laser scanning microscopy (CLSM) were also used to investigate vesicle penetration and drug entrapment in tissue cross-sections and in receiver medium. Vesicles were probed with 1,2-dihexadecanoyl-sn-glycero-3-phosphoethanolamine triethylammonium salt (Rh-PE) and fluorescein sodium $(\mathrm{NaFl})$ - which fluoresce red and green, respectively - to determine the penetration of ULs into tissue and receiver medium.

\section{Materials and methods}

\section{Materials}

Non-hydrogenated egg phosphatidylcholine (PC), Coatsome ${ }^{\circledR}$ NC-50, (with $\geq 95 \%$ purity) was obtained from NOF Corp (Tokyo, Japan). NaFl and d-limonene were purchased from Sigma-Aldrich Corp (St Louis, MO, USA). Tween ${ }^{\circledR} 20$ was obtained from Ajax Finechem Pty Ltd (Auckland, New Zealand). Lissamine ${ }^{\mathrm{TM}}$ rhodamine B (Rh-PE) and 4',6-diamino2-phenylindole dihydrochloride (DAPI) were purchased from Thermo Fisher Scientific (Waltham, MA, USA). Cholesterol (Chol) was obtained from Carlo Erba Reagents (Cornaredo, Italy). All other reagents were of analytical grade.

\section{Preparation of liposomes}

The UL formulation (consisting of PC, d-limonene, Chol, Tween 20, and NaFl) was prepared using a sonication method, as described in our previous study. ${ }^{20}$ Briefly, PC and
Chol were dissolved in a mixture of methanol and chloroform (1:2, by volume). The mixture of PC and Chol was dried, using nitrogen stream, until a thin, homogeneous film was created. The thin film obtained in a test tube was then stored for 6 hours in a desiccator connected to a vacuum pump. Afterwards, the dried thin lipid film was hydrated with phosphate buffered saline (PBS) and NaFl solution. Then, the hydrated lipid film was dispersed, using a sonicator bath, for 30 minutes. Tween 20 and d-limonene were added to the dispersion before sonication with a probe sonicator, for size reduction of the liposomes.

\section{Size and shape of the liposomes}

\section{Particle size and surface charge}

Distilled water was used to dilute the ULs. Then, the particle size, size distribution, and zeta potential were measured using a photon correlation spectroscopy particle size analyzer (Zetasizer Nano ZS, Malvern Instruments Ltd, Malvern, $\mathrm{UK}$ ) with a $4 \mathrm{~mW}$ helium-neon laser at a scattering angle of 173 degrees. All measurements were performed under ambient conditions, in triplicate.

\section{Transmission electron microscopy}

The ULs were observed using TEM for particle size and shape assessment. The sample was diluted with distilled water, then placed in sonicator bath for 10 minutes. The sample was then dropped onto a formvar-coated copper grid. The excess liposome sample on the copper grid was gently removed using filter paper. Afterwards, the sample was observed using an $80 \mathrm{kV}$ microscope (JEM 1230, JEOL Ltd, Tokyo, Japan).

\section{In vitro skin penetration study for TEM investigation}

\section{Skin preparation}

Abdominal porcine skin (obtained from intrapartum stillbirth animals provided by a farm in Nakhon Pathom) was used as a barrier membrane in this study. Subcutaneous fat was removed using surgical blades and scissors. The thickness of the abdominal skin was about $0.6-0.7 \mathrm{~mm}$. The prepared porcine skin was then stored in a refrigerator at $-20^{\circ} \mathrm{C}$ until use. Prior to the experiments, the skin was thawed using PBS at room temperature.

\section{Skin penetration study}

The skin penetration study was conducted using a $2.31 \mathrm{~cm}^{2}$ penetration area of Franz diffusion cells, connected 
to a circulating water bath. The temperature of the water bath was maintained at $32^{\circ} \mathrm{C}$. The porcine skin was mounted between the donor and receptor compartments of the diffusion chamber, with the stratum corneum facing the donor compartment. PBS was used as the receiver medium, filling the receiver compartment with $6.5 \mathrm{~mL}$. The receiver medium was stirred with a magnetic bar at a rate of $500 \mathrm{rpm}$. Two milliliters of ULs were placed into the donor compartment. The experiment was performed under occlusive conditions. At predetermined times of 30 minutes, 1 hour, 2 hours, and 4 hours, $0.5 \mathrm{~mL}$ of receiver medium was withdrawn for vesicle visualization, and the same volume of PBS was added to the receiver compartment, to maintain a constant volume. At the end of the skin penetration experiment, the skin samples were taken from the diffusion cells and washed with PBS. The skin samples were stored at $-20^{\circ} \mathrm{C}$ prior to TEM investigation.

\section{Skin preparation for TEM}

The treated skin samples were investigated for ultrastructural changes using TEM visualization. Intact skin was used as a control. The skin samples were cut into small pieces and fixed overnight (at $4^{\circ} \mathrm{C}$ ) with $2.5 \%$ glutaraldehyde (by volume), and $1 \%$ osmium tetroxide (weight to volume) in $0.1 \mathrm{M}$ PBS for 2 hours. After fixation, the samples were dehydrated in a range of ethanol solutions $(35 \%, 50 \%$, $70 \%, 95 \%$, and 100\%) and infiltrated with Spurr's resin. The resin-embedded samples were incubated at $70^{\circ} \mathrm{C}$ for 8 hours. Ultrathin sections were cut using an ultramicrotome (2088 Ultrotome V; LKB-Produkter AB, Bromma, Sweden) using a diamond knife (Ultra $45^{\circ}$, Diatome AG, Biel, Switzerland), collected on copper grids, and intensified with uranium acetate and lead citrate. The samples were visualized using TEM (JEM 1230). Ultrathick sections were cut by an ultramicrotome, stained with toluidine blue, and observed by light microscope.

\section{Receiver medium visualization by TEM}

The receiver medium was visualized to assess the penetration of vesicles through the skin - as intact or disintegrated vesicles. The receiver medium was filtered through a nylon membrane $(0.45 \mu \mathrm{m}$ pore size $)$ to remove contaminants. The filtrate was placed in a sonicator bath for 10 minutes before being dropped onto a formvar-coated copper grid. After drying, the sample was visualized under the microscope (JEM 1230) at $100 \mathrm{~K}$ magnification. Microscopy was performed at $80 \mathrm{kV}$.

\section{Confocal laser scanning microscopy study}

\section{Liposome preparation}

The fluorescent probe, Rh-PE, was dissolved in a mixture of methanol and chloroform (1:2, by volume) before being added to a test tube containing a mixture of PC and Chol. The ratio of $\mathrm{PC}$ to $\mathrm{Rh}-\mathrm{PE}$ was 100:1 M. The mixture of $\mathrm{Rh}-\mathrm{PE}, \mathrm{PC}$, and Chol was then prepared for thin film formation, according to the process described above.

\section{Separation of non-entrapped $\mathrm{NaFI}$ from ULs}

A filtration technique using Amicon ${ }^{\circledR}$ Ultra- 0.5 centrifugal devices (Millipore Corporation, Billerica, MA, USA) was utilized to separate non-entrapped drug from ULlabeled Rh-PE. The UL-labeled Rh-PE $(0.5 \mathrm{~mL})$ was added into an ultrafiltration tube, with a cut-off molecular weight of 3,000 Da, and then the mixture was centrifuged at $14,000 \times g$, at $4{ }^{\circ} \mathrm{C}$, for 30 minutes. The filtrate was removed, and then the retentate tube was inverted in a new collection tube, to collect the entrapped NaFl-loaded, ULlabeled Rh-PE via centrifugation at $1,000 \times g$, at $4^{\circ} \mathrm{C}$, for 2 minutes. The entrapped NaFl-loaded, UL-labeled Rh-PE obtained was immediately used in the skin penetration experiments.

\section{Skin penetration study}

The skin penetration of NaFl-loaded, UL-labeled Rh-PE was studied using the method described above. The donor compartment was filled with $150 \mu \mathrm{L}$ of the entrapped NaFlloaded, UL-labeled Rh-PE. The receiver medium and the treated skin samples were collected for analysis.

\section{Skin histology preparation}

The cross-sectional tissue samples of skin treated with NaFl-loaded, UL-labeled Rh-PE were prepared using a cryomicrotome (CM1850, Leica Microsystems GmbH, Wetzlar, Germany). Each treated skin sample was embedded in cryosection embedding medium (Neg 50 ${ }^{\mathrm{TM}}$, Microm International $\mathrm{GmbH}$, Waldorf, Germany) onto a metal sample holder inside a cryomicrotome, at $-30^{\circ} \mathrm{C}$. The embedded skin was sectioned into $10 \mu \mathrm{m}$ slices by microtome blades (Highprofile Disposable Blade 818, Leica Microsystems $\mathrm{GmbH}$, Wetzlar, Germany) before being placed onto adhesion slides (SuperFrost ${ }^{\circledR}$ Plus, Menzel-Gläser, Braunschweig, Germany). These sectioned tissues were stained with $3 \mu \mathrm{M}$ DAPI for 2 minutes (to stain the living cell layer of the skin), washed with PBS, mounted with mounting medium, and covered with a cover slip. 


\section{Receiver medium preparation}

The receiver medium was filtered through a nylon membrane $(0.45 \mu \mathrm{m}$ pore size) to remove any contaminants. The filtrate was dropped onto a glass slide and dried at room temperature. After drying, the sample was mounted with mounting medium and covered with a cover slip, prior to investigation with CLSM.

\section{CLSM imaging}

CLSM, using a colocalization technique, was employed to analyze the presence of NaFl-loaded, UL-labeled Rh-PE in the receiver medium, to elucidate the skin penetration route of ULs in tissue cross-sections. Different fluorescence colors of compounds among rhodamine-probed liposomes (red), $\mathrm{NaFl}$ as entrapped drug (green), and from living cells (blue) were applied to visualize the vesicle and drug distribution in the treated skin sample. The $10 \times$ objective lens of an inverted Zeiss LSM 510 Meta confocal microscope (Carl Zeiss Microscopy GmbH, Jena, Germany) equipped with a helium-neon laser (excitation wavelength: $543 \mathrm{~nm}$ ) for Rh-PE, an argon laser (excitation wavelength: $488 \mathrm{~nm}$ ) for $\mathrm{NaFl}$, and a diode laser (excitation wavelength: $358 \mathrm{~nm}$ ) for DAPI was used for receiver medium and cross-sectional tissue visualization. To investigate the possible skin penetration pathways of ULs, the mean fluorescence intensities from follicular and nonfollicular regions were compared. The fluorescence intensities obtained from the middle vertical line of each image were calculated using Zeiss LSM 5 operating software.

\section{Data analysis and statistics}

Statistical significance of differences was examined using the Student's $t$-test. The significance level was set at $P<0.05$.

\section{Results and discussion Size and shape of ULs}

The size, shape, and surface charge of ULs were measured using a particle size analyzer. The average size of ULs was $37.23 \pm 0.2 \mathrm{~nm}$, with a narrow size distribution (polydispersity index: 0.22). The zeta potential was negative $(-10.6 \pm 0.65 \mathrm{mV})$. The zeta potential of liposomes composed of PC and Chol only was $-3.78 \pm 0.11 \mathrm{mV}$ (data not shown). $\mathrm{NaFI}$ is an anionic compound. PC is a zwitterionic compound, with an isoelectric point (pl) between 6-6.7. ${ }^{21}$ The $\mathrm{pH}$ in our experimental conditions $(\mathrm{pH}:$ 7.4) was higher than the pI. Therefore, the PC vesicles had negative charge; therefore, NaFI-loaded ULs exhibited a negative charge.
A TEM image of the ULs (Figure 1) shows that UL vesicles were nanospheres. The average size of ULs, as determined by TEM, was $34.08 \pm 2.96 \mathrm{~nm}$. The average size of ULs obtained from dynamic light scattering and TEM techniques was not significantly different. A small UL particle size is desired for vesicle penetration of skin. ${ }^{22}$

\section{Mechanism of enhanced skin penetration TEM study of treated skin}

The prepared skin samples were observed via TEM, to evaluate the mechanism of enhanced penetration by ULs. The ultrathick sections of intact skin and skin treated with ULs, observed via light microscopy, are shown in Figure 2. Figure 2A shows intact skin; Figures $2 \mathrm{~B}$ and $2 \mathrm{C}$ show regions of follicular and nonfollicular skin, respectively, treated with ULs, at 4 hours. The stratum corneum of intact skin (Figure 2A) was normal. In the treated skin, it was detached (Figures 2B and 2C).

Figure 3 shows TEM images of intact skin. Figures $3 \mathrm{~A}$ and $3 \mathrm{~B}$ show an overview of the stratum corneum and stratum granulosum in the epidermis. In Figure 3C, intercellular lipid lamellae were observed in the intercellular space as electron lucent bands. Corneocytes were visualized by their characteristic flattened shape, absence of organelles, and presence of electron-dense keratin filaments. Moreover, corneodesmosomes were observed, connecting corneocytes between layers of the stratum corneum. Figure 3D shows corneodesmosomes connecting cornified cells in the stratum corneum to one another, as well as to keratinocyte cells in the stratum granulosum.

Figure 4 shows TEM images of ultrathick sections of porcine skin treated with ULs (from Figure 2B).

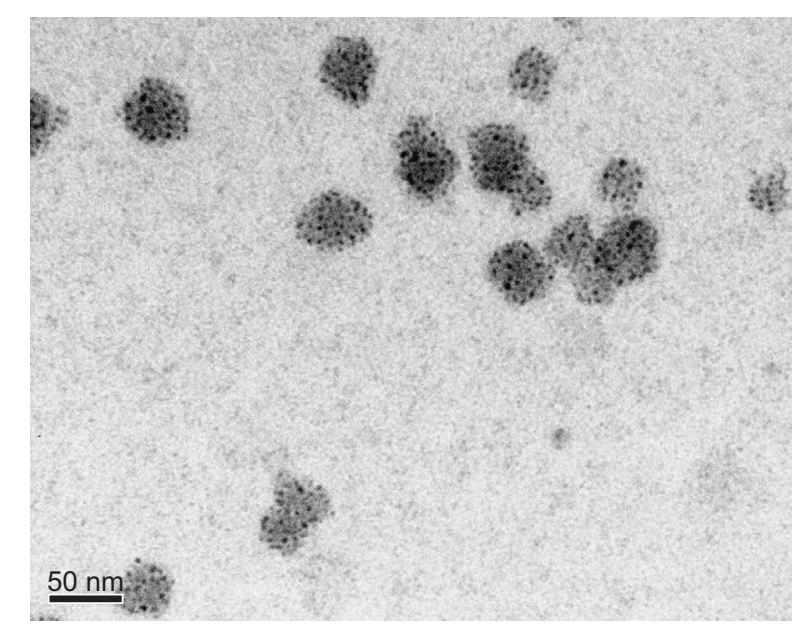

Figure I Transmission electron microscopy images of ultradeformable liposomes. Notes: Magnification: $200 \mathrm{~K}$. Scale bar represents $50 \mathrm{~nm}$. 
A

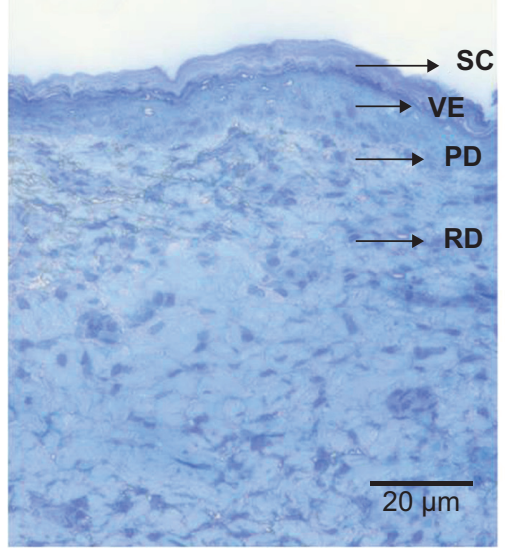

B

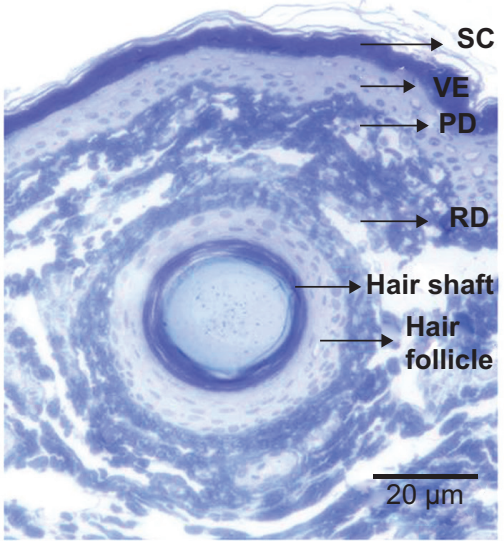

C

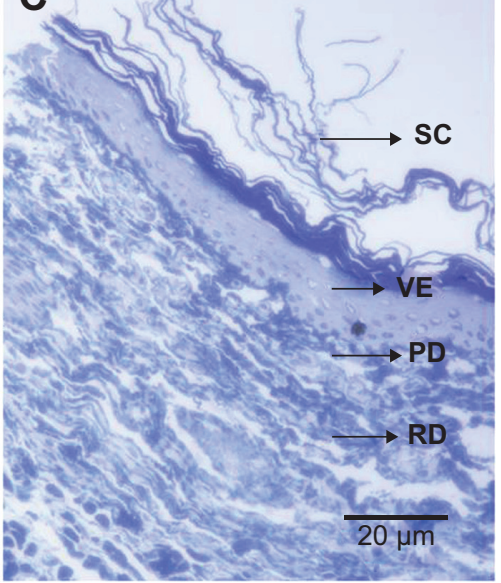

Figure 2 Ultrathick sections observed by light microscopy.

Notes: (A) Intact pig skin, (B) and (C) pig skin treated with ultradeformable liposomes at 4 hours, from follicular and nonfollicular regions, respectively. Each scale bar represents $20 \mu \mathrm{m}$.

Abbreviations: SC, stratum corneum; VE, viable epidermis; PD, papillary dermis; RD, reticular dermis.

Figures $4 \mathrm{~A}$ and $4 \mathrm{~B}$ show the upper and lower stratum corneum, respectively, at the same magnification. The upper stratum corneum (Figure 4A) had broader-shaped corneocytes than in the lower stratum corneum (Figure 4B).
Figure 4C shows that porcine skin treated with ULs had obviously less electron-dense keratin filaments, compared against intact skin (Figure 3C). The broad shape and the fewer electron-dense keratin filaments of the corneocytes in
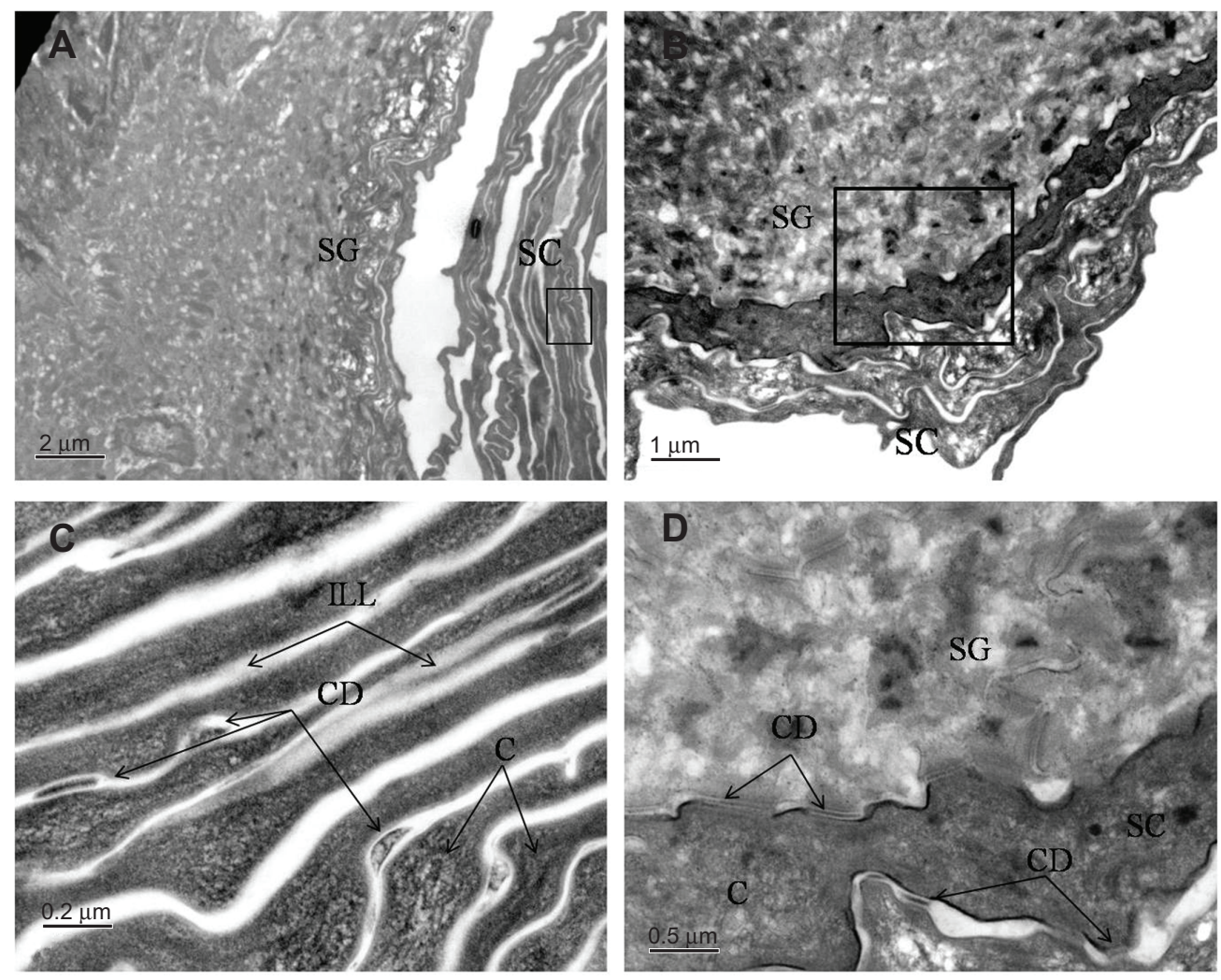

Figure 3 Transmission electron microscopy images of intact pig skin.

Notes: (A) Overview of SC and SG; scale bar represents $2 \mu \mathrm{m}$. (B) Overview of SC and SG; scale bar represents I $\mu \mathrm{m}$. (C) Magnification of marked area in (A); scale bar represents $0.2 \mu \mathrm{m}$. (D) Magnification of marked area in (B); scale bar represents $0.5 \mu \mathrm{m}$.

Abbreviations: SC, stratum corneum; SG, stratum granulosum; C, corneocyte; ILL, intercellular lipid lamellae; CD, corneodesmosome. 

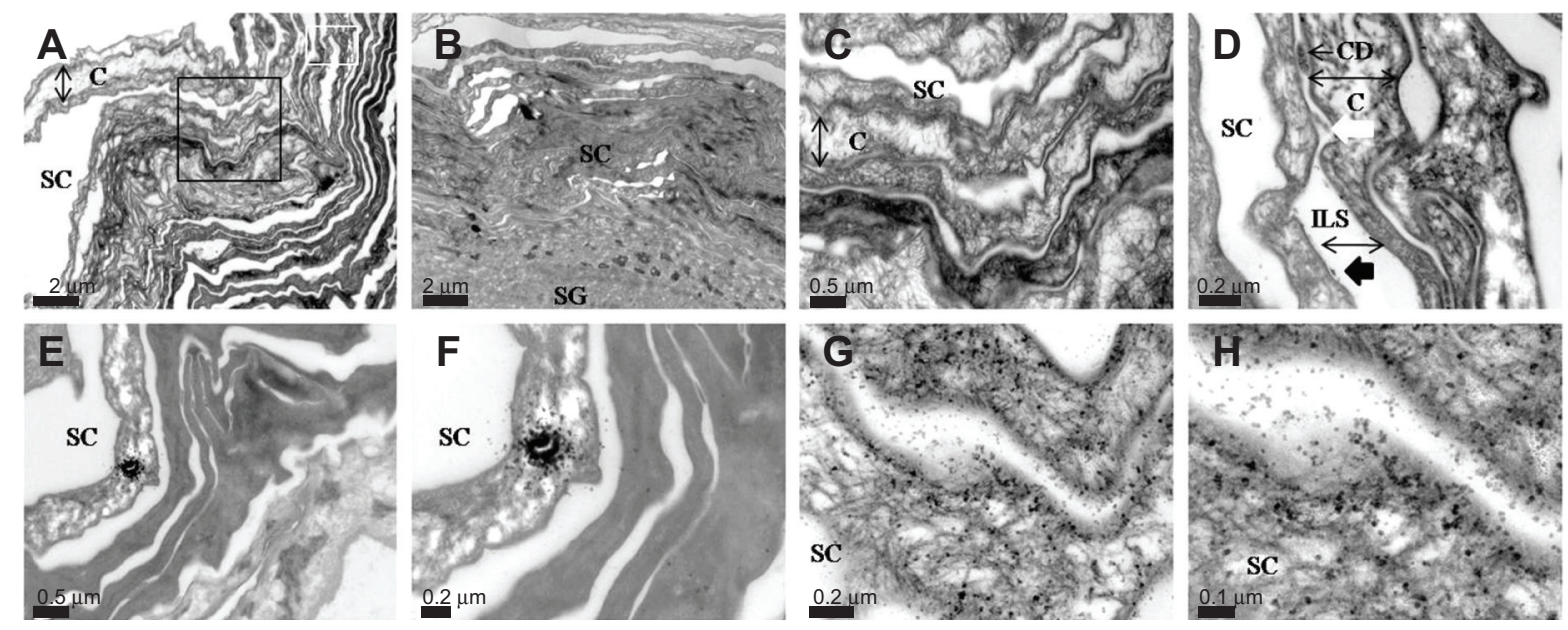

Figure 4 Transmission electron microscopy images of pig skin treated with ultradeformable liposomes obtained from ultrathick section of pig skin treated with ultradeformable liposomes at 4 hours.

Notes: (A) Corneocytes in SC; scale bar represents $2 \mu \mathrm{m}$. (B) SC and SG; scale bar represents $2 \mu \mathrm{m}$. (C) Magnification of area in (A) marked with black rectangle; scale bar represents $0.5 \mu \mathrm{m}$. (D) Magnification of area in (A) marked with white rectangle; black arrow indicates degraded corneodesmosome; white arrow indicates degrading corneodesmosome in SC; scale bar represents $0.2 \mu \mathrm{m}$. (E) Vesicle penetration into corneocyte; scale bar represents $0.5 \mu \mathrm{m}$. (F) Magnification of (E); scale bar represents $0.2 \mu \mathrm{m}$. (G) Vesicle fragmentation in SC; scale bar represents $0.2 \mu \mathrm{m}$. (H) Magnification of (G); scale bar represents $0.1 \mu \mathrm{m}$.

Abbreviations: SC, stratum corneum; SG, stratum granulosum; C, corneocyte; CD, corneodesmosome; ILS, intercellular space.

the stratum corneum resulted from denaturation of keratin inside the corneocytes. At the same magnification, the intercellular spaces of porcine skin treated with ULs (Figure 4D) were wider than those of intact skin (Figure 3C), because the corneodesmosomes that connected the corneocytes between each stratum corneum layer were degraded. As a result of the degradation of corneodesmosomes, the stratum corneum layers of treated skin were detached, as seen in ultrathick sections (Figures 2B and 2C). Since the hydration effect of PBS resulted in corneocyte swelling and intercellular lipid lamellae disruption, ${ }^{23,24}$ the detachment of the stratum corneum layers in Figures 2B and 2C might result from both liposome-induced corneodesmosome degradation and the hydration effect. Some corneodesmosomes are present in the upper part of Figure 4D, whereas the lower part shows degrading (white arrow) and degraded (black arrow) corneodesmosomes. These images indicate that the degradation of corneodesmosomes depends on the rate at which ULs penetrate the skin.

Figure 4E shows a UL particle and denatured keratin inside a corneocyte. When observed at higher magnification (Figure 4F), it was obvious that the particle was disintegrating into smaller pieces. Figures $4 \mathrm{G}$ and $4 \mathrm{H}$ show many disintegrated particles inside corneocytes and in the intercellular space of the stratum corneum. Keratin inside corneocytes was denatured, and no corneodesmosomes were observed. Figures 4E and 4F reveal that when particles penetrated the corneocyte, they gradually disintegrated into smaller pieces of phospholipid bilayer, denatured keratin, and disrupted corneodesmosomes - thereby enhancing the penetration of entrapped drugs. Figures $4 \mathrm{G}$ and $4 \mathrm{H}$ also show particle fragmentation to be mostly confined to the inner cellular membrane and intercellular lipid of corneocytes. Because the inside of corneocytes contains keratin, while inner and outer cellular membranes of corneocytes are lipid and protein, respectively, most of the lipid vesicles were confined to the inner cellular envelope and extracellular lipid matrix.

Figure 5 shows TEM images of ultrathick sections of porcine skin treated with ULs (from Figure 2C). Figures 5A and $5 \mathrm{~B}$ show the degraded corneodesmosomes at the interface of the stratum corneum and stratum granulosum (black arrow). In Figure 5B, disintegrated particles were observed near the desmosomes. In viable epidermis (Figure 5C), desmosomes and hemidesmosomes, which connect stratum basale and papillary dermis (Figure 5D), were normal. From Figures 5A through 5D, it is apparent that only corneodesmosomes were degraded, whereas the desmosomes in viable epidermis and hemidesmosomes in the epidermis-dermis interface were normal. Desmosomes are intercellular junctions that maintain tissue integrity of the epidermis. The main components of desmosomes are cadherins, armadillo proteins, and plakins. Corneodesmosomes are cellular junctions, derived from desmosomes, that provide strong intercorneocyte cohesion within the stratum corneum. The extracellular proteins of corneodesmosomes, which mediate corneocyte cohesion, are desmoglein 1, desmocollin 1, and corneodesmosin - a secreted glycoprotein that is incorporated into desmosomes prior to their conversion to corneodesmosomes in the 

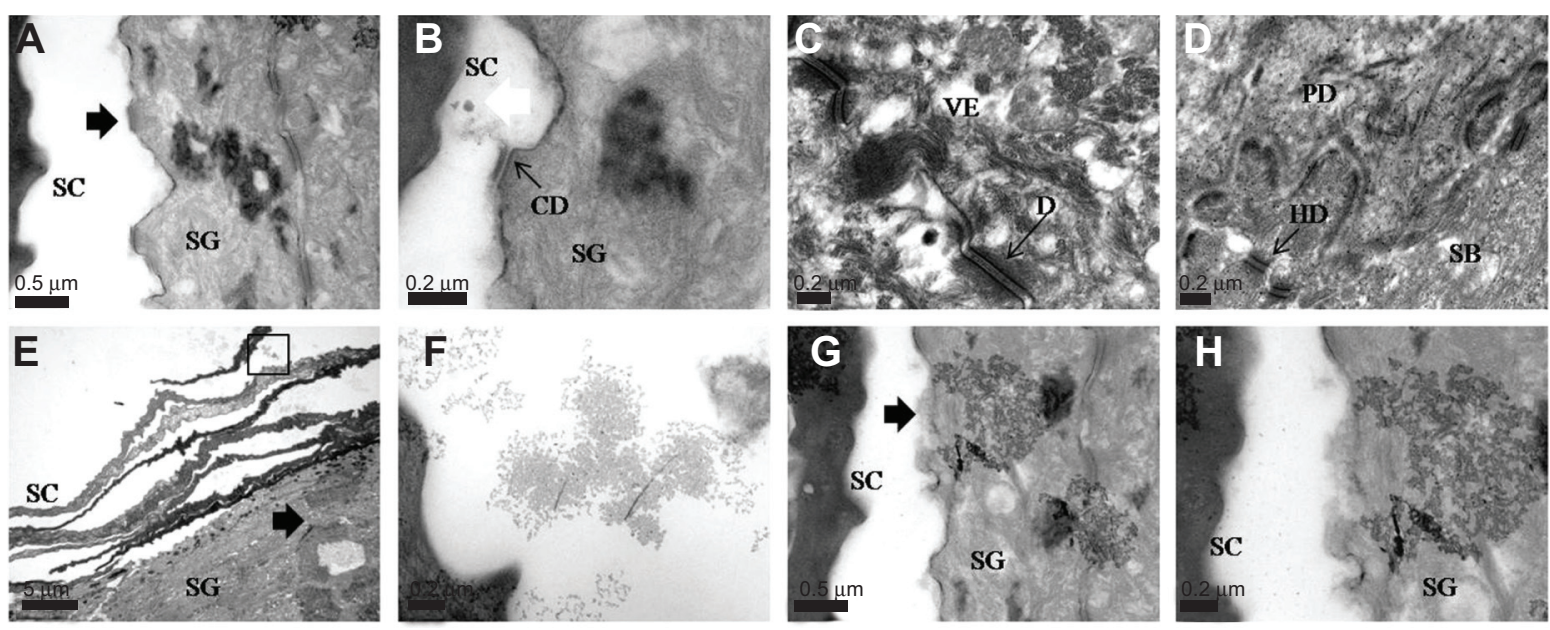

Figure 5 Transmission electron microscopy images of pig skin treated with ultradeformable liposomes, obtained from ultrathick section of pig skin treated with ultradeformable liposomes at 4 hours.

Notes: (A) Corneodesmosome degradation (black arrow) between SC and SG; scale bar represents $0.5 \mu$ m. (B) Corneodesmosome degradation (black arrow) and disintegrated particle (white arrow) between SC and SG; scale bar represents $0.2 \mu \mathrm{m}$. (C) Desmosome in viable epidermis; scale bar represents $0.2 \mu \mathrm{m}$. (D) Hemidesmosome at stratum basale and papillary dermis; scale bar represents $0.2 \mu \mathrm{m}$. (E) Ultradeformable liposome penetration into the skin (black arrow); scale bar represents $5 \mu \mathrm{m}$. (F) Magnification of marked area in (E); scale bar represents $0.2 \mu \mathrm{m}$. (G) Disrupted cell membrane (black arrow) from penetration of intact vesicles into SG; scale bar represents $0.5 \mu \mathrm{m}$. (H) Magnification of (G); scale bar represents $0.2 \mu \mathrm{m}$.

Abbreviations: SC, stratum corneum; SG, stratum granulosum; SB, stratum basale; PD, papillary dermis; D, desmosome; CD, corneodesmosome; HD, hemidesmosome; VE, viable epidermis.

stratum corneum. ${ }^{25,26}$ We suggest that corneodesmosomes are degraded by their interaction with UL vesicles. Other desmosomes and hemidesmosomes are unaffected, which may be due to the difference in desmosomal protein structures between the stratum corneum and viable epidermis, as mentioned above.

Figure 5E shows the penetration of ULs through the stratum corneum into viable epidermis. The area of penetration shows obvious detachment of the stratum corneum, which resulted from corneodesmosome degradation. In the upper portion of the penetrated area, intact vesicles were observed, as shown in Figure 5F. Several upper stratum corneum layers were loosened, due to a desquamation process. As a result, intact vesicles were found in the intercellular region.

The presence of intact vesicles in the stratum granulosum (Figures $5 \mathrm{G}$ and $5 \mathrm{H}$ ) indicates that ULs can penetrate viable epidermis as intact vesicles. The uppermost keratinocyte membrane in the stratum granulosum was disrupted by the penetration of ULs (Figures $5 \mathrm{G}$ and $5 \mathrm{H}$ ). The presence of intact vesicles found in viable epidermis may be caused by the denaturing of keratin inside corneocytes and the degradation of corneodesmosomes by interactions with UL vesicles.

From our TEM images (Figures 4 and 5), it is apparent that ULs increase drug penetration by acting as a penetration enhancer and drug carrier system. The ULs enhanced skin penetration by entering corneocytes before disintegrating into small fragments and interacting with the protein and lipid barrier structure of the stratum corneum. The vesicles denatured keratin filaments inside corneocytes and degraded corneodesmosomes, resulting in vesicle and drug penetration into viable epidermis and dermis. Intracellular proteins and protein structures may become denatured as a result of their interactions with the surfactants in vesicles (Tween 20 and phospholipid). ${ }^{27,28} \mathrm{~d}$-limonene is a monoterpene skin penetration enhancer, incorporated in ULs, that could break interlamellar hydrogen bonding within the lipid bilayer. This interaction leads to widening of the aqueous region near polar head groups, and results in increased diffusion of polar molecules. ${ }^{29}$ Our findings also support the lipid protein partitioning theory, which proposes that penetration enhancers act by disrupting intercellular lipid organization, modifying intracellular proteins, resulting in increased drug penetration into viable epidermis. ${ }^{30}$

A distinct difference between follicular and nonfollicular regions of skin interacting with vesicles was that there were more vesicle fragments found in the stratum corneum of the follicular region, as shown in Figures $4 \mathrm{G}$ and $4 \mathrm{H}$. The presence of more vesicle fragments in the follicular region, as observed by TEM, confirmed our previous suggestion that the follicular pathway was the main permeation pathway of ULs. ${ }^{21}$

\section{Receiver medium observation and CLSM study}

The collected receiver medium was investigated using TEM and CLSM, to determine the mechanism of the drug carrier system. 
TEM was used to investigate the receiver medium from TEM visualization, at time points of 30 minutes, 1 hour, 2 hours, and 4 hours; those images are shown in Figures 6A through 6D, respectively. The receiver medium showed the spherical shape and nanoscale of vesicles, indicating that ULs could penetrate through skin to the receiver medium as intact vesicles.

Figures 7A through 7D show CLSM images of receiver medium at time points of 30 minutes, 1 hour, 2 hours, and 4 hours. The presence of red fluorescence and green fluorescence in the receiver medium indicated that both ULs and $\mathrm{NaFl}$ could penetrate through skin. The ULs found in the receiver medium could be both intact vesicles and phospholipid bilayer. Regarding the presence of intact vesicles in the receiver medium, as observed by TEM (Figures 6A-6D), NaFl found in the receiver medium could be both entrapped drug and free drug. The presence of both colors of fluorescence at the same point could also be used to investigate the release behavior of ULs. It is assumed that red fluorescence in receiver medium is intact vesicle. If the green fluorescence intensity is less than the red fluorescence intensity, the entrapped drug was released. Inside the white circles of Figures 7A-1, 7A-3, and $7 \mathrm{~A}-4$, the green fluorescence intensity was markedly less than the red fluorescence intensity, indicating that the vesicles had released some part of the entrapped drug into the skin. If both the green fluorescence and red fluorescence appeared equal, the entrapped drug was not released, or a significant amount was not released. In Figures 7B-1, 7B-3, 7B-4, 7C-1, 7C-3, and 7C-4, both green fluorescence and red fluorescence were equal, indicating that the entrapped drug was not released, or a significant amount was not released. If green fluorescence appears while red fluorescence does not, there is free drug. Inside the white circles of Figures 7D-1, 7D-3, and 7D-4, green fluorescence appeared, whereas red fluorescence did not appear, indicating there was free drug in the receiver medium. Supported by the presence of intact vesicles in the receiver medium (Figures 6 and 7), we suggest that ULs might penetrate through skin to the systemic blood circulation as intact vesicles. Pegylated or stealth liposomes should be applied for transdermal drug delivery, instead of conventional liposomes, to reduce reticuloendothelial system uptake and to prolong circulation time in blood.

Using CLSM and size exclusion chromatography, Cevc et $\mathrm{al}^{15}$ compared the vesicle size of phospholipid ULs from
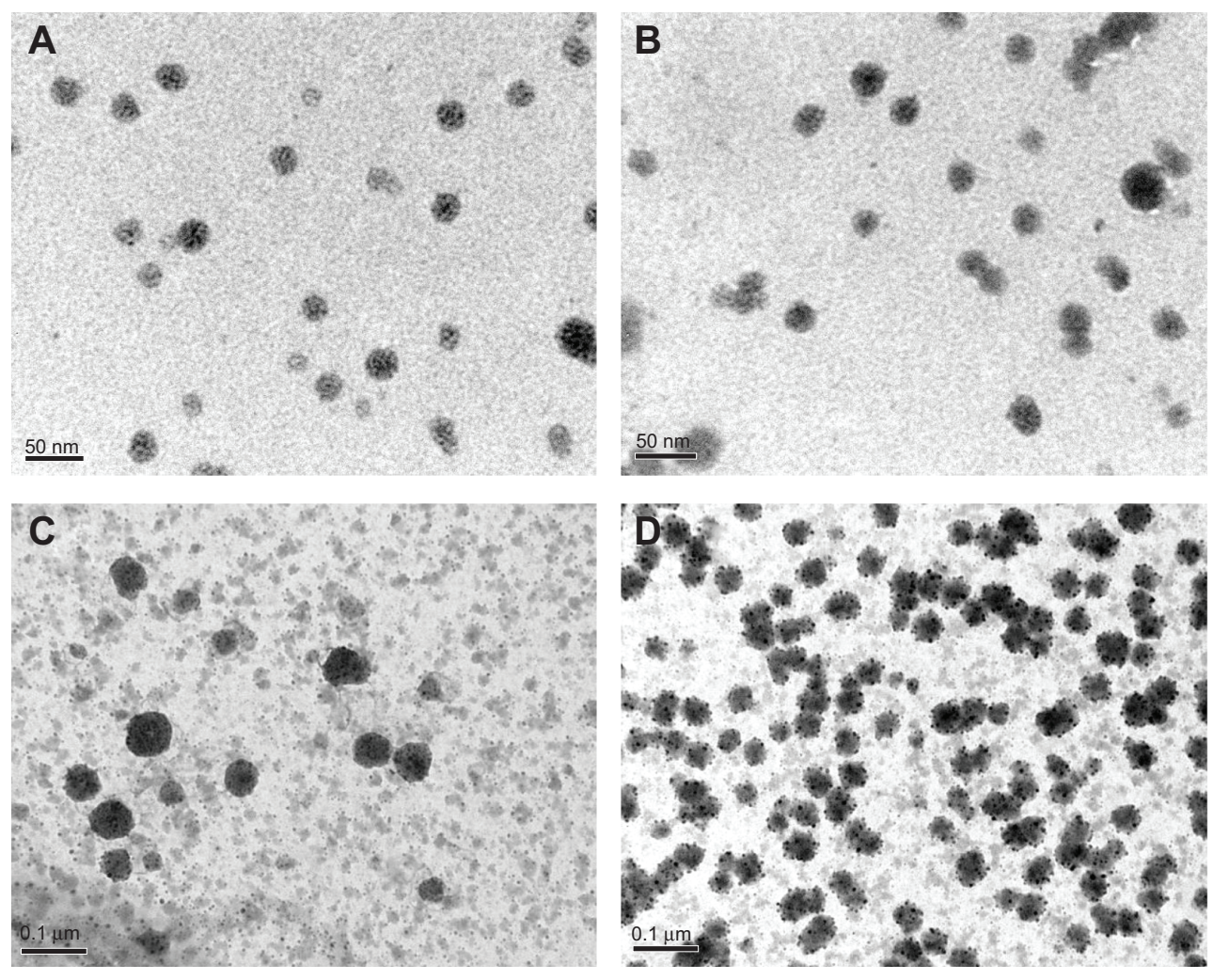

Figure 6 Transmission electron microscopy images of the receiver medium after skin penetration study of the NaFl-loaded, Rh-PE-labeled ultradeformable liposomes at different time points.

Notes: (A) 30 minutes; scale bar represents $50 \mathrm{~nm}$. (B) I hour; scale bar represents $50 \mathrm{~nm}$. (C) 2 hours; scale bar represents I00 nm. (D) 4 hours; scale bar represents I00 nm. Abbreviations: $\mathrm{NaFl}$, fluorescein sodium; Rh-PE, rhodamine B I,2-dihexadecanoyl-sn-glycero-3-phosphoethanolamine triethylammonium salt. 

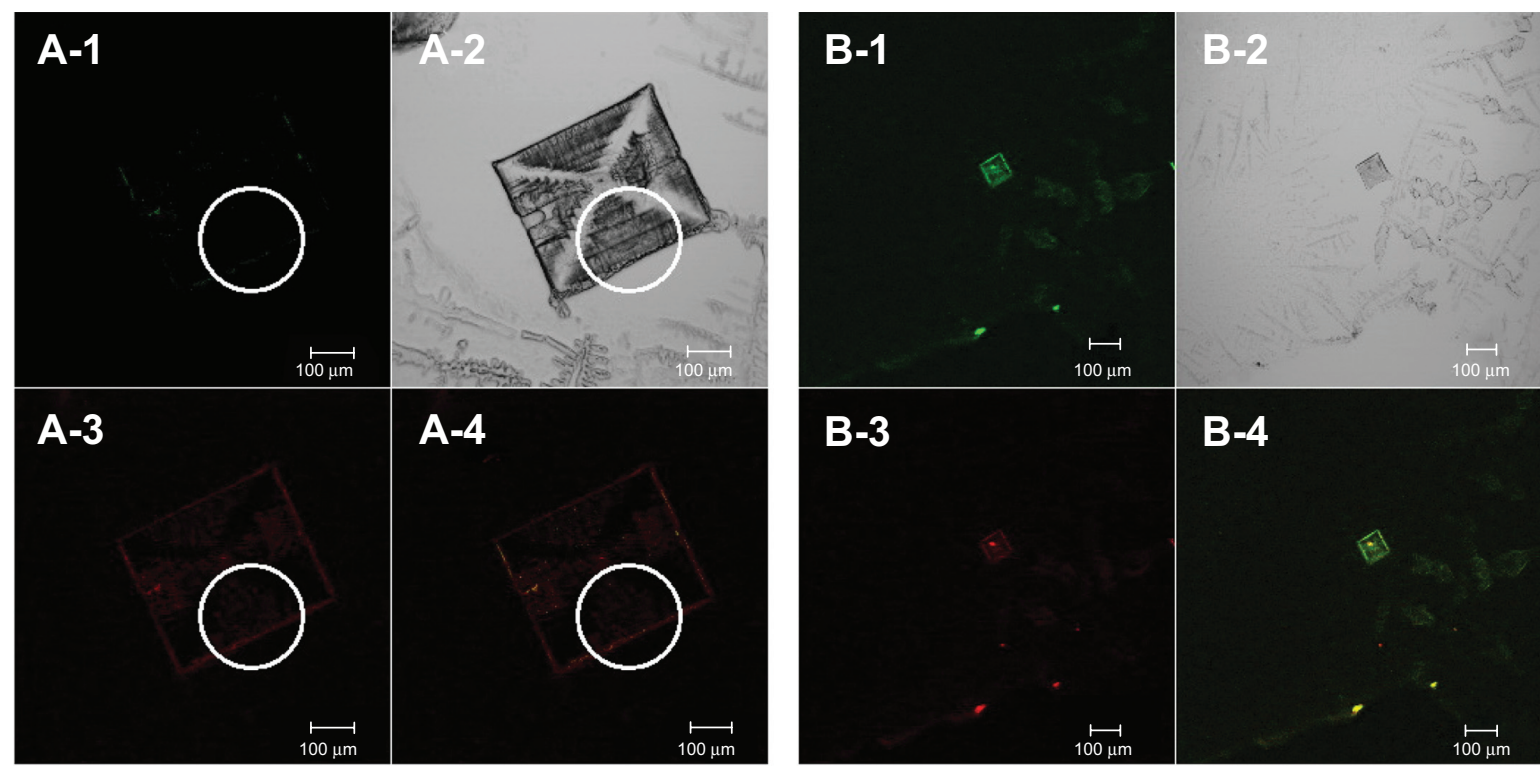

B-4
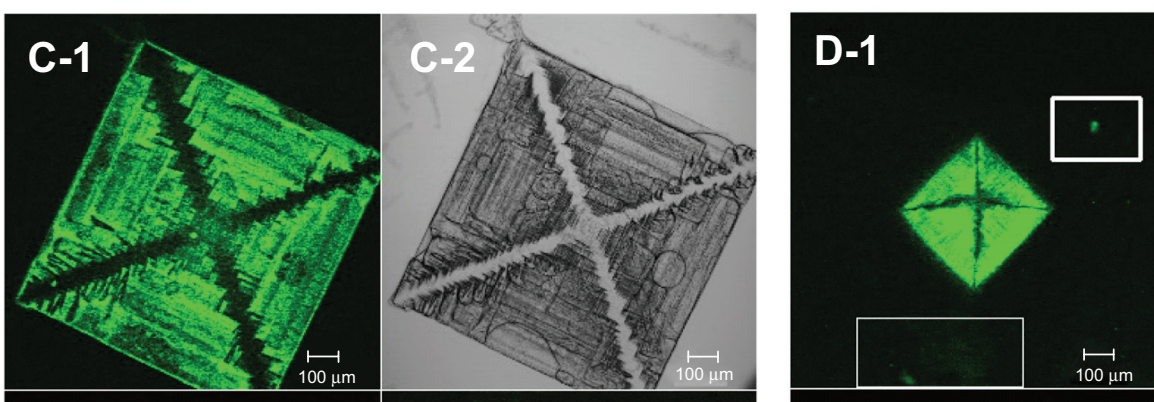

D-2
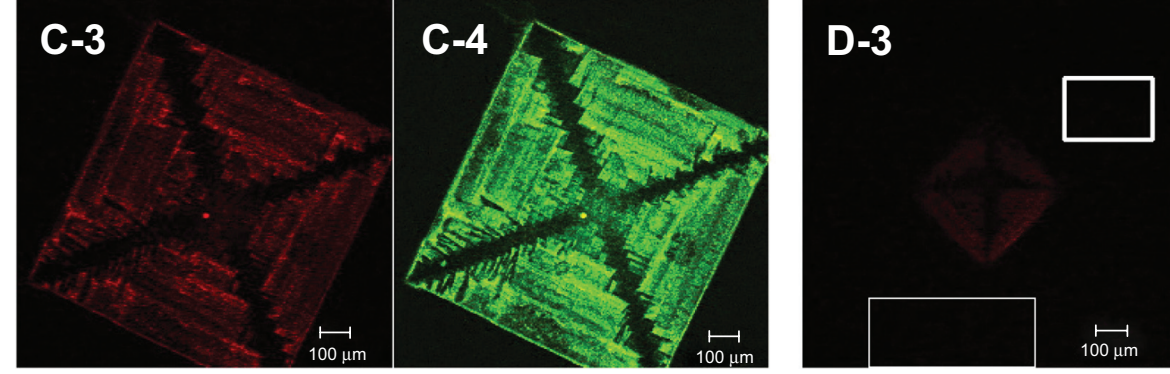

D-4

Figure 7 Confocal laser scanning microscopy images of receiver medium from the skin penetration study of NaFl-loaded, Rh-PE-labeled ultradeformable liposomes at different time points.

Notes: (A) 30 minutes; (B) I hour; (C) 2 hours; (D) 4 hours. Each image is divided into four parts: -I, green fluorescence of NaFl; -2, bright field image; -3, red fluorescence of Rh-PE; -4 , overlay of -1 and $\mathbf{- 3}$. Scale bars represent $100 \mu \mathrm{m}$.

Abbreviations: NaFl, fluorescein sodium; Rh-PE, rhodamine B I,2-dihexadecanoyl-sn-glycero-3-phosphoethanolamine triethylammonium salt.

their original preparation with those collected from the serum of mice who had the ULs applied to their skin. The size of vesicles from the original preparation did not differ from those obtained from the mouse serum. The authors concluded that ULs penetrated through the skin to the circulation system as intact vesicles, without permanent disintegration. Therefore, based on our TEM images of intact vesicles found in viable epidermis (Figures 5G and 5H) and receiver medium (Figures 6A through 6D), as well as CLSM images of receiver medium (Figures 7A through 7D), we agreed with Cevc et $\mathrm{al}^{7}$ - that ULs could penetrate the skin and enter the blood circulation system as intact vesicles. Honeywell-Nguyen et $\mathrm{al}^{16,17}$ studied in vivo and in vitro interactions between nonionic elastic vesicles and human skin, using tape stripping techniques. TEM images revealed that little vesicle material was found in the deepest layers of the stratum corneum. Thus, the authors suggested that penetration of intact vesicles into viable epidermis might not be possible. Because the specimen used for TEM preparation was a very small piece, the probability of finding intact vesicles in viable epidermis was also very small. To confirm the penetration of intact vesicles into viable epidermis or into the blood circulation system, 

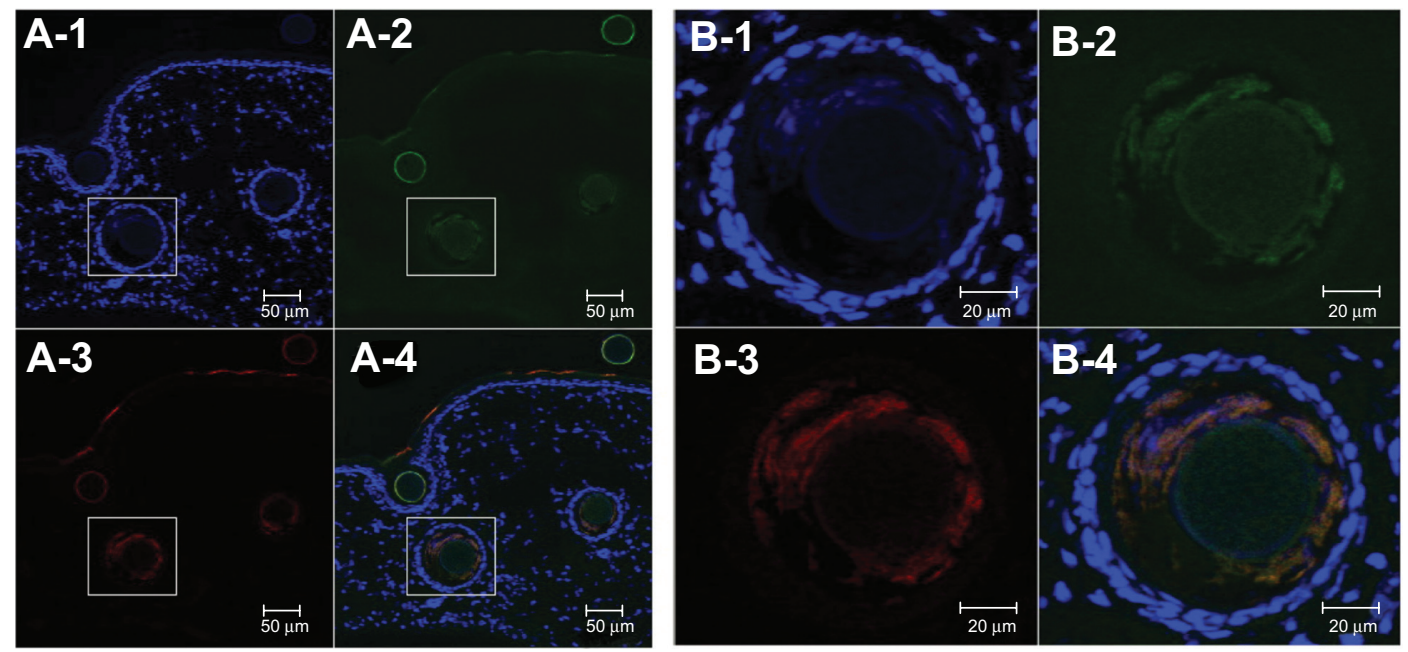

Figure 8 Confocal laser scanning microscopy cross-sectional images of porcine skin treated with NaFl-loaded, Rh-PE-labeled ultradeformable liposomes, at 2 hours.

Notes: (A) Scale bars represent $50 \mu \mathrm{m}$. (B) Magnification of marked area in (A); scale bars represent $20 \mu \mathrm{m}$. Each image is divided into four parts: - I, blue fluorescence of $\mathrm{DAPI}, \mathbf{- 2}$, green fluorescence of $\mathrm{NaFl} ; \mathbf{- 3}$, red fluorescence of $\mathrm{Rh}-\mathrm{PE} ; \mathbf{- 4}$, overlay of $\mathbf{- 1}, \mathbf{- 2}$, and $\mathbf{- 3}$.

Abbreviations: NaFl, fluorescein sodium; Rh-PE, rhodamine B I,2-dihexadecanoyl-sn-glycero-3-phosphoethanolamine triethylammonium salt; DAPI, 4',6-diamidino-2phenylindole.

the receiver medium (or collected serum) also should be investigated.

Figures $8 \mathrm{~A}$ and $8 \mathrm{~B}$ show cross-sections of skin treated with NaFl-loaded, Rh-PE-labeled ULs at 2 hours. Both green fluorescence and red fluorescence were seen throughout the tissue cross-section, with high-intensity deposits in the follicular region, indicating that $\mathrm{NaFl}$ and $\mathrm{ULs}$ could permeate from the stratum corneum to the dermis and accumulate in the hair follicles, more than in other regions, as shown in
Figures $8 \mathrm{~A}-2$, 8A-3, and 8A-4. Figures 8B-2, 8B-3, and $8 \mathrm{~B}-4$ show the hair follicle at higher magnification. The orange fluorescence inside the hair follicle, seen in the merged image (Figure 8B-4), occurred due to green fluorescence and red fluorescence at the same region, which indicated that $\mathrm{NaFl}$ was still entrapped in ULs when it had penetrated via the hair follicles.

Sequential cross-sectional images of follicular and nonfollicular regions of porcine skin treated with NaFl-loaded,
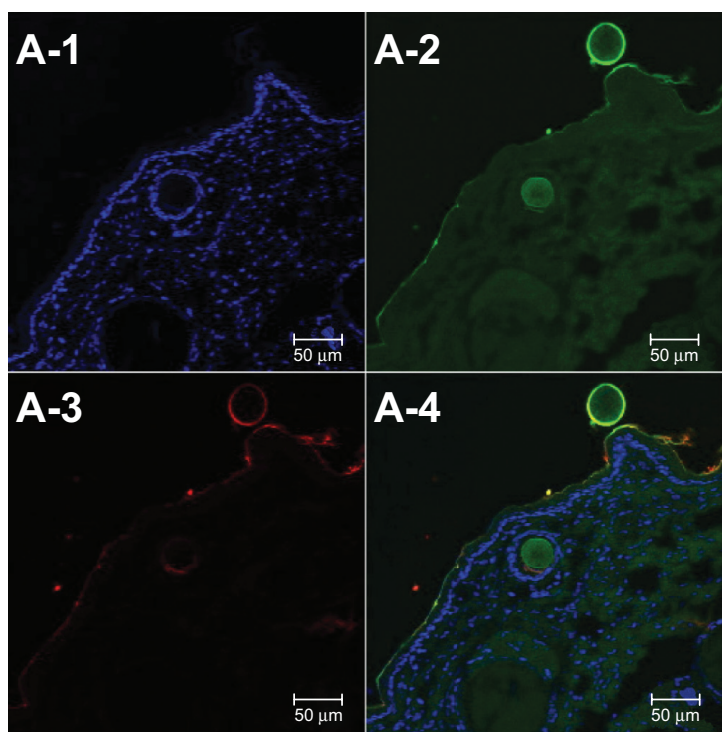

\section{A-4}

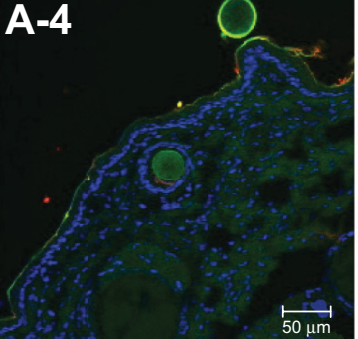

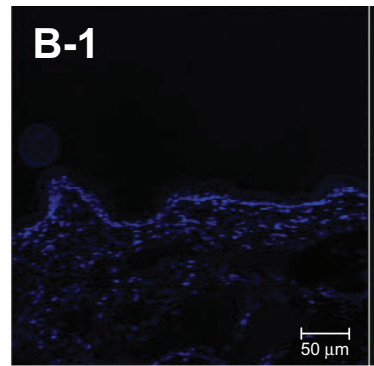
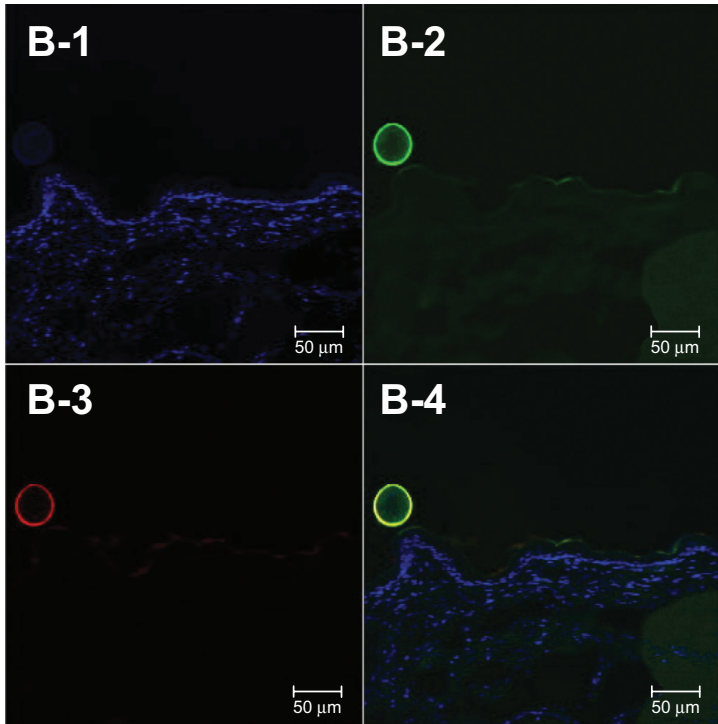

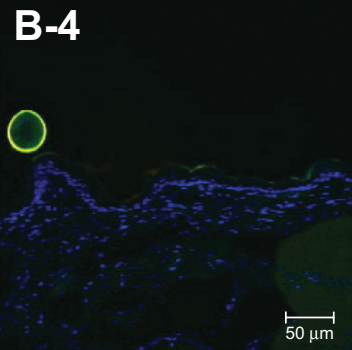

Figure 9 Confocal laser scanning microscopy cross-sectional images of porcine skin treated with NaFl-loaded Rh-PE-labeled ULs and stained with DAPI, at 4 hours.

Notes: (A) Follicular region; (B) nonfollicular region. Each image is divided into four parts: - I, blue fluorescence of DAPI; -2, green fluorescence of NaFl; -3, red fluorescence of Rh-PE; -4, overlay of $-1,-2$, and $\mathbf{- 3}$. Scale bar represents $50 \mu \mathrm{m}$.

Abbreviations: ULs, ultradeformable liposomes; NaFl, fluorescein sodium; Rh-PE, rhodamine B I,2-dihexadecanoyl-sn-glycero-3-phosphoethanolamine triethylammonium salt; DAPI, 4',6-diamidino-2-phenylindole. 
Table I Fluorescence intensities of $\mathrm{NaFl}$ and Rh-PE at follicular and nonfollicular regions

\begin{tabular}{lll}
\hline Region & NaFI & Rh-PE \\
\hline Follicular region & $425.60 \pm 210.07 *$ & $103.12 \pm 57.78 *$ \\
Nonfollicular region & $255.01 \pm 61.43$ & $80.41 \pm 29.37$ \\
\hline
\end{tabular}

Notes: $* P<0.05$, compared against nonfollicular region. Each value represents the mean \pm standard deviation.

Abbreviations: $\mathrm{NaFl}$, fluorescein sodium; Rh-PE, rhodamine B I,2-dihexadecanoylsn-glycero-3-phosphoethanolamine triethylammonium salt.

Rh-PE-labeled ULs (at 4 hours) are shown in Figures 9A and 9B. Both green fluorescence and red fluorescence intensities at follicular regions (Figures 9A-2 and 9A-3) were significantly higher than at nonfollicular regions (Figures 9B-2 and 9B-3) (Table 1), indicating that NaFl and ULs penetrated via the follicular region more than via the nonfollicular region.

Theoretically, molecules permeate the skin through intercellular, intracellular (transcellular), and follicular (transappendageal) pathways. ${ }^{31}$ Follicular channels offer a large space for drug and vesicle transportation, bypassing the barrier function of the stratum corneum. The interaction of vesicle and intracellular protein in the transcellular pathway resulted in vesicle fragmentation, as shown in Figures 4E through 4H. Regarding our TEM and CLSM investigation, we suggest that the intact vesicles found in receiver medium might penetrate skin using a transappendageal pathway as the main transportation route.

\section{Conclusion}

Our TEM investigation revealed that ULs employed both a penetration-enhancing effect and a drug carrier system mechanism for enhancement of skin penetration. ULs act as penetration enhancers by denaturing intracellular protein and corneodesmosomes, and disrupting intercellular lipid organization, which results in enhancement of penetration via intracellular and intercellular pathways, respectively. ULs also act as a drug carrier system, by penetrating through skin into receiver medium as intact vesicles, as observed by TEM and CLSM. We suggest that a transappendageal pathway is the major penetration route of intact vesicles.

\section{Acknowledgments}

The authors are thankful for the financial support of Thailand Research Funds, through the Golden Jubilee PhD Program (Grant number: PHD/0057/2551), and the TRF Basic Research Grant (Grant number: BRG 5680016).

\section{Disclosure}

The authors report no conflicts of interest in this work.

\section{References}

1. Wang Y, Thakur R, Fan Q, Michniak B. Transdermal iontophoresis: combination strategies to improve transdermal iontophoretic drug delivery. Eur J Pharm Biopharm. 2005;60(2):179-191.

2. Herwadkar A, Sachdeva V, Taylor LF, Silver H, Banga AK. Low frequency sonophoresis mediated transdermal and intradermal delivery of ketoprofen. Int J Pharm. 2012;423(2):289-296.

3. Kolli CS, Banga AK. Characterization of solid maltose microneedles and their use for transdermal delivery. Pharm Res. 2008;25(1):104-113.

4. Shah KA, Date AA, Joshi MD, Patravale VB. Solid lipid nanoparticles (SLN) of tretinoin: potential in topical delivery. Int $J$ Pharm. 2007;345(1-2):163-171.

5. Dragicevic-Curic N, Scheglmann D, Albrecht V, Fahr A. Temoporfinloaded invasomes: development, characterization and in vitro skin penetration studies. J Control Release. 2008;127(1):59-69.

6. Cevc G, Blume G. Lipid vesicles penetrate into intact skin owing to the transdermal osmotic gradients and hydration force. Biochim Biophys Acta. 1992;1104(1):226-232.

7. Cevc G, Gebauer D, Stieber J, Schätzlein A, Blume G. Ultraflexible vesicles, Transfersomes, have an extremely low pore penetration resistance and transport therapeutic amounts of insulin across the intact mammalian skin. Biochim Biophys Acta. 1998;1368(2):201-215.

8. Cevc G. Transdermal drug delivery of insulin with ultradeformable carriers. Clin Pharmacokinet. 2003;42(5):461-474.

9. Mishra D, Dubey V, Asthana A, Saraf DK, Jain MK. Elastic liposomes mediated transcutaneous immunization against Hepatitis B. Vaccine. 2006;24(22):4847-4855.

10. Cevc G, Mazgareanu S, Rother M. Preclinical characterization of NSAIDs in ultradeformable carriers or conventional topical gels. Int $J$ Pharm. 2008;360(1-2):29-39.

11. Rother M, Seidel EJ, Clarkson PM, Mazgareanu S, Vierl U, Rother I. Efficacy of epicutaneous Diractin (ketoprofen in Transfersome gel) for the treatment of pain related to eccentric muscle contractions. Drug Des Devel Ther. 2009;3:143-149.

12. El Maghraby GM, Williams AC, Barry BW. Skin delivery of oestradiol from deformable and traditional liposomes: mechanistic studies. J Pharm Pharmacol. 1999;51(1):1123-1134.

13. van den Bergh BA, Bouwstra JA, Junginger HE, Wertz PW. Elasticity of vesicles affects hairless mouse skin structure and permeability. J Control Release. 1999;62(3):367-379.

14. Cevc G, Blume G. New, highly efficient formulation of diclofenac for the topical, transdermal administration in ultradeformable drug carriers, Transfersomes. Biochim Biophys Acta. 2001;1514(2):191-205.

15. Cevc G, Schätzlein A, Richardsen H. Ultradeformable lipid vesicles can penetrate the skin and other semi-permeable barriers unfragmented. Evidence from double label CLSM experiments and direct size measurements. Biochim Biophys Acta. 2002;1564(1):21-30.

16. Honeywell-Nguyen PL, de Graaff AM, Groenink HW, Bouwstra JA. The in vivo and in vitro interactions of elastic and rigid vesicles with human skin. Biochim Biophys Acta. 2002;1573(2):130-140.

17. Honeywell-Nguyen PL, Bouwstra JA. The in vitro transport of pergolide from surfactant-based elastic vesicles through human skin: a suggested mechanism of action. J Control Release. 2003;86(1):145-156.

18. Honeywell-Nguyen PL, Bouwstra JA. Vesicles as a tool for transdermal and dermal delivery. Drug Discov Today. 2005;2:67-74.

19. Subongkot T, Duangjit S, Rojanarata T, Opanasopit $P$, Ngawhirunpat T. Ultradeformable liposomes with terpenes for delivery of hydrophilic compound. J Liposome Res. 2012;22(3):254-262.

20. Subongkot T, Wonglertnirant N, Songprakhon P, Rojanarata T, Opanasopit $P$, Ngawhirunpat $T$. Visualization of ultradeformable liposomes penetration pathways and their skin interaction by confocal laser scanning microscopy. Int J Pharm. 2013;441(1-2):151-161.

21. Chain E, Kemp I. The isoelectric points of lecithin and sphingomyelin. Biochem J. 1934;28(6):2052-2055.

22. Verma DD, Verma S, Blume G, Fahr A. Particle size of liposomes influences dermal delivery of substances into skin. Int J Pharm. 2003 258(1-2):141-151. 
23. Warner RR, Stone KJ, Boissy YL. Hydration disrupts human stratum corneum ultrastucture. J Invest Dermatol. 2003;120(3):275-284.

24. Bouwstra JA, de Graaff A, Gooris GS, Nijsse J, Wiechers JW, van Aelst AC. Water distribution and related morphology in human stratum corneum at different hydration levels. J Invest Dermatol. 2003;120(5): $750-758$.

25. Caubet C, Jonca N, Brattsand M, et al. Degradation of corneodesmosome proteins by two serine proteases of the kallikrein family, SCTE/ KLK5/hK5 and SCCE/KLK7/hK7. J Invest Dermatol. 2004;122(5): 1235-1244.

26. Garrod D, Chidgey M. Desmosome structure, composition and function. Biochim Biophys Acta. 2008;1778(3):572-587.
27. Williams AC, Barry BW. Penetration enhancers. Adv Drug Deliv Rev. 2004;56(5):603-618.

28. Som I, Bhatia K, Yasir M. Status of surfactants as penetration enhancers in transdermal drug delivery. J Pharm Bioallied Sci. 2012;4(1):2-9.

29. Narishetty ST, Panchagnula R. Transdermal delivery of zidovudine: effect of terpenes and their mechanism of action. $J$ Control Release. 2004;95(3):367-379.

30. Barry BW. Lipid-Protein-Partitioning theory of skin penetration enhancement. J Control Release. 1991;15:237-248.

31. Barry BW. Novel mechanisms and devices to enable successful transdermal drug delivery. Eur J Pharm Sci. 2001;14(2):101-114.

\section{Publish your work in this journal}

The International Journal of Nanomedicine is an international, peerreviewed journal focusing on the application of nanotechnology in diagnostics, therapeutics, and drug delivery systems throughout the biomedical field. This journal is indexed on PubMed Central, MedLine, CAS, SciSearch $®$, Current Contents ${ }^{\circledR} /$ Clinical Medicine,
Journal Citation Reports/Science Edition, EMBase, Scopus and the Elsevier Bibliographic databases. The manuscript management system is completely online and includes a very quick and fair peer-review system, which is all easy to use. Visit http://www.dovepress.com/ testimonials.php to read real quotes from published authors. 